Article

\title{
Inextensible Flows of Curves on Lightlike Surfaces
}

\author{
Zühal Küçükarslan Yüzbaşı ${ }^{1}$ and Dae Won Yoon ${ }^{2, * \mathbb{D}}$ \\ 1 Department of Mathematics, Firat University, Elazig 23119, Turkey; zuhal2387@yahoo.com.tr \\ 2 Department of Mathematics Education and RINS, Gyeongsang National University, Jinju 52828, Korea \\ * Correspondence: dwyoon@gnu.ac.kr; Tel.: +82-55-772-2256
}

Received: 28 September 2018; Accepted: 26 October 2018; Published: 29 October 2018

check for updates

\begin{abstract}
In this paper, we study inextensible flows of a curve on a lightlike surface in Minkowski three-space and give a necessary and sufficient condition for inextensible flows of the curve as a partial differential equation involving the curvatures of the curve on a lightlike surface. Finally, we classify lightlike ruled surfaces in Minkowski three-space and characterize an inextensible evolution of a lightlike curve on a lightlike tangent developable surface.
\end{abstract}

Keywords: inextensible flow; lightlike surface; ruled surface; Darboux frame

\section{Introduction}

It is well known that many nonlinear phenomena in physics, chemistry and biology are described by dynamics of shapes, such as curves and surfaces, and the time evolution of a curve and a surface has significance in computer vision and image processing. The time evolution of a curve and a surface is described by flows, in particular inextensible flows of a curve and a surface. Physically, inextensible flows give rise to motion, for which no strain energy is induced. The swinging motion of a cord of fixed length or of a piece of paper carried by the wind can be described by inextensible flows of a curve and a surface. Furthermore, the flows arise in the context of many problems in computer vision and computer animation [1-4].

Chirikjian and Burdick [1] studied applications of inextensible flows of a curve. In [5], the authors derived the time evolution equations for an inextensible flow of a space curve and also studied inextensible flows of a developable ruled surface. In [6], the author investigated the general description of the binormal motion of a spacelike and a timelike curve in a three-dimensional de Sitter space and gave some explicit examples of a binormal motion of the curves. Schief and Rogers [4] studied the binormal motions of curves with constant curvature and torsion. Many authors have studied geometric flow problems [7-11].

The outline of the paper is organized as follows: In Section 2, we give some geometric concepts in Minkowski space and present the pseudo-Darboux frames of a spacelike curve and a lightlike curve on a lightlike surface. In Sections 3 and 4, we study inextensible flows of a spacelike curve and a lightlike curve on a lightlike surface. In the last section, we classify lightlike ruled surfaces and study inextensible flows of lightlike tangent developable surfaces.

\section{Preliminaries}

The Minkowski three-space $\mathbb{R}_{1}^{3}$ is a real space $\mathbb{R}^{3}$ with the indefinite inner product $\langle\cdot, \cdot\rangle$ defined on each tangent space by:

$$
\langle\mathbf{x}, \mathbf{y}\rangle=-x_{0} y_{0}+x_{1} y_{1}+x_{2} y_{2},
$$

where $\mathbf{x}=\left(x_{0}, x_{1}, x_{2}\right)$ and $\mathbf{y}=\left(y_{0}, y_{1}, y_{2}\right)$ are vectors in $\mathbb{R}_{1}^{3}$.

A nonzero vector $\mathbf{x}$ in $\mathbb{R}_{1}^{3}$ is said to be spacelike, timelike or lightlike if $\langle\mathbf{x}, \mathbf{x}\rangle>0,\langle\mathbf{x}, \mathbf{x}\rangle<0$ or $\langle\mathbf{x}, \mathbf{x}\rangle=0$, respectively. Similarly, an arbitrary curve $\gamma=\gamma(s)$ is spacelike, timelike or lightlike if all of 
its tangent vectors $\gamma^{\prime}(s)$ are spacelike, timelike or lightlike, respectively. Here "prime" denotes the derivative with respect to the parameter $s$.

Let $M$ be a lightlike surface in Minkowski three-space $\mathbb{R}_{1}^{3}$, that is the induced metric of $M$ is degenerate. Then, a curve $\gamma$ on $M$ is spacelike or lightlike.

Case 1: If $\gamma$ is a spacelike curve, we can reparametrize it by the arc length $s$. Therefore, we have the unit tangent vector $\mathbf{t}(s)=\gamma^{\prime}(s)$ of $\gamma(s)$. Since $M$ is a lightlike surface, we have a lightlike normal vector $\mathbf{n}$ along $\gamma$. Therefore, we can choose a vector $\mathbf{g}$ satisfying:

$$
\langle\mathbf{n}, \mathbf{g}\rangle=1, \quad\langle\mathbf{t}, \mathbf{g}\rangle=\langle\mathbf{g}, \mathbf{g}\rangle=0 .
$$

Then, we have pseudo-orthonormal frames $\{\mathbf{t}, \mathbf{n}, \mathbf{g}\}$, which are called the Darboux frames along $\gamma(s)$. By standard arguments, we have the following Frenet formulae:

$$
\frac{d}{d s}\left(\begin{array}{c}
\mathbf{t}(s) \\
\mathbf{n}(s) \\
\mathbf{g}(s)
\end{array}\right)=\left(\begin{array}{ccc}
0 & \kappa_{g}(s) & \kappa_{n}(s) \\
-\kappa_{n}(s) & \tau_{g}(s) & 0 \\
-\kappa_{g}(s) & 0 & -\tau_{g}(s)
\end{array}\right)\left(\begin{array}{c}
\mathbf{t}(s) \\
\mathbf{n}(s) \\
\mathbf{g}(s)
\end{array}\right)
$$

where $\kappa_{n}=\left\langle\mathbf{t}^{\prime}(s), \mathbf{n}(s)\right\rangle, \kappa_{g}=\left\langle\mathbf{t}^{\prime}(s), \mathbf{g}(s)\right\rangle$ and $\tau_{g}=-\left\langle\mathbf{n}(s), \mathbf{g}^{\prime}(s)\right\rangle$.

Case 2: Let $\gamma$ be a lightlike curve parametrized by a pseudo arc length parameter $s$ on a lightlike surface $M$ in $\mathbb{R}_{1}^{3}$. Since a normal vector $\mathbf{n}$ of a lightlike surface $M$ is lightlike, we can choose a vector $\mathbf{g}$ such that:

$$
\langle\mathbf{g}, \mathbf{g}\rangle=1, \quad\langle\mathbf{t}, \mathbf{g}\rangle=\langle\mathbf{g}, \mathbf{n}\rangle=0 .
$$

Furthermore, we consider:

$$
\langle\mathbf{t}, \mathbf{n}\rangle=1 \text {. }
$$

Then, we have pseudo-orthonormal Darboux frames $\{\mathbf{t}, \mathbf{n}, \mathbf{g}\}$ along a nongeodesic lightlike curve $\gamma(s)$ on $M$ and get the following Frenet formulae:

$$
\frac{d}{d s}\left(\begin{array}{c}
\mathbf{t}(s) \\
\mathbf{n}(s) \\
\mathbf{g}(s)
\end{array}\right)=\left(\begin{array}{ccc}
\kappa_{n}(s) & 0 & \kappa_{g}(s) \\
0 & -\kappa_{n}(s) & \tau_{g}(s) \\
-\tau_{g}(s) & -\kappa_{g}(s) & 0
\end{array}\right)\left(\begin{array}{c}
\mathbf{t}(s) \\
\mathbf{n}(s) \\
\mathbf{g}(s)
\end{array}\right)
$$

where $\kappa_{n}=\left\langle\mathbf{t}^{\prime}(s), \mathbf{n}(s)\right\rangle, \kappa_{g}=\left\langle\mathbf{t}^{\prime}(s), \mathbf{g}(s)\right\rangle$ and $\tau_{g}=-\left\langle\mathbf{n}(s), \mathbf{g}^{\prime}(s)\right\rangle$.

\section{Inextensible Flows of a Spacelike Curve}

We assume that $\gamma:[0, l] \times[0, w] \rightarrow M \subset \mathbb{R}_{1}^{3}$ is a one-parameter family of the smooth spacelike curve on a lightlike surface in $\mathbb{R}_{1}^{3}$, where $l$ is the arc length of the initial curve. Let $u$ be the curve parametrization variable, $0 \leq u \leq l$. We put $v=\left\|\frac{\partial \gamma}{\partial u}\right\|$, from which the arc length of $\gamma$ is defined by $s(u)=\int_{0}^{u} v d u$. Furthermore, the operator $\frac{\partial}{\partial s}$ is given in terms of $u$ by $\frac{\partial}{\partial s}=\frac{1}{v} \frac{\partial}{\partial u}$, and the arc length parameter is given by $d s=v d u$.

On the Darboux frames $\{\mathbf{t}, \mathbf{n}, \mathbf{g}\}$ of the spacelike curve $\gamma$ on a lightlike surface $M$ in $\mathbb{R}_{1}^{3}$, any flow of $\gamma$ can be given by:

$$
\frac{\partial \gamma}{\partial t}=f_{1} \mathbf{t}+f_{2} \mathbf{n}+f_{3} \mathbf{g}
$$

where $f_{1}, f_{2}, f_{3}$ are scalar speeds of the spacelike curve $\gamma$ on a lightlike surface $M$, respectively. We put $s(u, t)=\int_{0}^{u} v d u$; it is called the arc length variation of $\gamma$. From this, the requirement that the curve is not subject to any elongation or compression can be expressed by the condition:

$$
\frac{\partial}{\partial t} s(u, t)=\int_{0}^{u} \frac{\partial v}{\partial t} d u=0
$$

for all $u \in[0, l]$. 
Definition 1. A curve evolution $\gamma(u, t)$ and its flow $\frac{\partial \gamma}{\partial t}$ of a spacelike curve in $\mathbb{R}_{1}^{3}$ are said to be inextensible if:

$$
\frac{\partial}{\partial t}\left\|\frac{\partial \gamma}{\partial u}\right\|=0
$$

Now, we give the arc length preserving condition for curve flows.

Theorem 1. Let $M$ be a lightlike surface in Minkowski three-space $\mathbb{R}_{1}^{3}$ and $\{\mathbf{t}, \mathbf{n}, \mathbf{g}\}$ be the Darboux frames of a spacelike curve $\gamma$ on $M$. If $\frac{\partial \gamma}{\partial t}=f_{1} \mathbf{t}+f_{2} \mathbf{n}+f_{3} \mathbf{g}$ is a flow of $\gamma$ on a lightlike surface $M$ in $\mathbb{R}_{1}^{3}$, then we have the following equation:

$$
\frac{\partial v}{\partial t}=\frac{\partial f_{1}}{\partial u}-v f_{2} \kappa_{n}-v f_{3} \kappa_{g}
$$

Proof. From the definition of a spacelike curve $\gamma$, we have $v^{2}=\left\langle\frac{\partial \gamma}{\partial u}, \frac{\partial \gamma}{\partial u}\right\rangle$. Since $u$ and $t$ are independent coordinates, $\frac{\partial}{\partial u}$ and $\frac{\partial}{\partial t}$ commute. Therefore, by differentiating $v^{2}$, we have:

$$
\begin{aligned}
2 v \frac{\partial v}{\partial t} & =\frac{\partial}{\partial t}\left\langle\frac{\partial \gamma}{\partial u}, \frac{\partial \gamma}{\partial u}\right\rangle \\
& =2\left\langle\frac{\partial \gamma}{\partial u}, \frac{\partial}{\partial u}\left(\frac{\partial \gamma}{\partial t}\right)\right\rangle \\
& =2\left\langle\frac{\partial \gamma}{\partial u}, \frac{\partial}{\partial u}\left(f_{1} \mathbf{t}+f_{2} \mathbf{n}+f_{3} \mathbf{g}\right)\right\rangle \\
& =2 v\left\langle\mathbf{t},\left(\frac{\partial f_{1}}{\partial u}-v f_{2} \kappa_{n}-v f_{3} \kappa_{g}\right) \mathbf{t}+\left(\frac{\partial f_{2}}{\partial u}+v f_{1} \kappa_{g}+v f_{2} \tau_{g}\right) \mathbf{n}+\left(\frac{\partial f_{3}}{\partial u}+v f_{1} \kappa_{n}-v f_{3} \tau_{g}\right) \mathbf{g}\right\rangle \\
& =2 v\left(\frac{\partial f_{1}}{\partial u}-v f_{2} \kappa_{n}-v f_{3} \kappa_{g}\right)
\end{aligned}
$$

This completes the proof.

Corollary 1. Let $\frac{\partial \gamma}{\partial t}=f_{1} \mathbf{t}+f_{2} \mathbf{n}+f_{3} \mathbf{g}$ be a flow of a spacelike curve $\gamma$ on a lightlike surface $M$ in $\mathbb{R}_{1}^{3}$. If the curve $\gamma$ is a geodesic curve or an asymptotic curve, then the following equation holds, respectively:

$$
\frac{\partial v}{\partial t}=\frac{\partial f_{1}}{\partial u}-v f_{2} \kappa_{n}
$$

or:

$$
\frac{\partial v}{\partial t}=\frac{\partial f_{1}}{\partial u}-v f_{3} \kappa_{g}
$$

Theorem 2. (Necessary and sufficient condition for an inextensible flow)

Let $\frac{\partial \gamma}{\partial t}=f_{1} \mathbf{t}+f_{2} \mathbf{n}+f_{3} \mathbf{g}$ be a flow of a spacelike curve $\gamma$ on a lightlike surface $M$ in $\mathbb{R}_{1}^{3}$. Then, the flow is inextensible if and only if:

$$
\frac{\partial f_{1}}{\partial s}=f_{2} \kappa_{n}+f_{3} \kappa_{g}
$$

Proof. Suppose that the flow of a spacelike curve $\gamma$ on $M$ is inextensible. From (4) and (5), we have:

$$
\frac{\partial}{\partial t} s(u, t)=\int_{0}^{u} \frac{\partial v}{\partial t} d u=\int_{0}^{u}\left(\frac{\partial f_{1}}{\partial u}-v f_{2} \kappa_{n}-v f_{3} \kappa_{g}\right) d u=0
$$

It follows that:

$$
\frac{\partial f_{1}}{\partial u}=v f_{2} \kappa_{n}+v f_{3} \kappa_{g}
$$

Since $\frac{\partial}{\partial s}=\frac{1}{v} \frac{\partial}{\partial u}$, we can obtain (6).

Conversely, by following a similar way as above, the proof is completed. 
Theorem 3. Let $\frac{\partial \gamma}{\partial t}=f_{1} \mathbf{t}+f_{2} \mathbf{n}+f_{3} \mathbf{g}$ be a flow of a spacelike curve $\gamma$ on a lightlike surface $M$ in $\mathbb{R}_{1}^{3}$. If the flow is inextensible, then a time evolution of the Darboux frame $\{\mathbf{t}, \mathbf{n}, \mathbf{g}\}$ along a curve $\gamma$ on a lightlike surface $M$ is given by:

$$
\frac{d}{d t}\left(\begin{array}{c}
\mathbf{t} \\
\mathbf{n} \\
\mathbf{g}
\end{array}\right)=\left(\begin{array}{ccc}
0 & \varphi_{1} & \varphi_{2} \\
-\varphi_{2} & \varphi_{3} & 0 \\
-\varphi_{1} & 0 & -\varphi_{3}
\end{array}\right)\left(\begin{array}{l}
\mathbf{t} \\
\mathbf{n} \\
\mathbf{g}
\end{array}\right)
$$

where:

$$
\begin{aligned}
& \varphi_{1}=\frac{\partial f_{2}}{\partial s}+f_{1} \kappa_{g}+f_{2} \tau_{g}, \\
& \varphi_{2}=\frac{\partial f_{3}}{\partial s}+f_{1} \kappa_{n}-f_{3} \tau_{g}, \\
& \varphi_{3}=\left\langle\frac{\partial \mathbf{n}}{\partial t}, \mathbf{g}\right\rangle .
\end{aligned}
$$

Proof. Noting that:

$$
\begin{aligned}
\frac{\partial \mathbf{t}}{\partial t} & =\frac{\partial}{\partial t}\left(\frac{\partial \gamma}{\partial s}\right)=\frac{\partial}{\partial s}\left(f_{1} \mathbf{t}+f_{2} \mathbf{n}+f_{3} \mathbf{g}\right) \\
& =\left(\frac{\partial f_{2}}{\partial s}+f_{1} \kappa_{g}+f_{2} \tau_{g}\right) \mathbf{n}+\left(\frac{\partial f_{3}}{\partial s}+f_{1} \kappa_{n}-f_{3} \tau_{g}\right) \mathbf{g}
\end{aligned}
$$

On the other hand,

$$
\begin{aligned}
& 0=\frac{\partial}{\partial t}\langle\mathbf{t}, \mathbf{n}\rangle=\left\langle\frac{\partial \mathbf{t}}{\partial t}, \mathbf{n}\right\rangle+\left\langle\mathbf{t}, \frac{\partial \mathbf{n}}{\partial t}\right\rangle=\frac{\partial f_{3}}{\partial s}+f_{1} \kappa_{n}-f_{3} \tau_{g}+\left\langle\mathbf{t}, \frac{\partial \mathbf{n}}{\partial t}\right\rangle \\
& 0=\frac{\partial}{\partial t}\langle\mathbf{t}, \mathbf{g}\rangle=\left\langle\frac{\partial \mathbf{t}}{\partial t}, \mathbf{g}\right\rangle+\left\langle\mathbf{t}, \frac{\partial \mathbf{g}}{\partial t}\right\rangle=\frac{\partial f_{2}}{\partial s}+f_{1} \kappa_{g}+f_{2} \tau_{g}+\left\langle\mathbf{t}, \frac{\partial \mathbf{n}}{\partial t}\right\rangle
\end{aligned}
$$

because of $\langle\mathbf{n}, \mathbf{n}\rangle=\langle\mathbf{g}, \mathbf{g}\rangle=0$ and $\langle\mathbf{n}, \mathbf{g}\rangle=1$.

Thus, we have:

$$
\begin{aligned}
& \frac{\partial \mathbf{n}}{\partial t}=-\left(\frac{\partial f_{3}}{\partial s}+f_{1} \kappa_{n}-f_{3} \tau_{g}\right) \mathbf{t}+\varphi_{3} \mathbf{n}, \\
& \frac{\partial \mathbf{g}}{\partial t}=-\left(\frac{\partial f_{2}}{\partial s}+f_{1} \kappa_{g}+f_{2} \tau_{g}\right) \mathbf{t}-\varphi_{3} \mathbf{g},
\end{aligned}
$$

where $\varphi_{3}=\left\langle\frac{\partial \mathbf{n}}{\partial t}, \mathbf{g}\right\rangle$. This completes the proof.

Now, by using Theorem 3, we give the time evolution equations of the geodesic curvature, the normal curvature and the geodesic torsion of a spacelike curve on a lightlike surface.

Theorem 4. Let $\frac{\partial \gamma}{\partial t}=f_{1} \mathbf{t}+f_{2} \mathbf{n}+f_{3} \mathbf{g}$ be a flow of a spacelike curve $\gamma$ on a lightlike surface $M$ in $\mathbb{R}_{1}^{3}$. Then, the time evolution equations of the functions $\kappa_{g}, \kappa_{n}$ and $\tau_{g}$ for the inextensible spacelike curve $\gamma$ are given by:

$$
\begin{aligned}
& \frac{\partial \kappa_{g}}{\partial t}=\frac{\partial \varphi_{1}}{\partial s}+\varphi_{1} \tau_{g}-\varphi_{3} \kappa_{g}, \\
& \frac{\partial \kappa_{n}}{\partial t}=\frac{\partial \varphi_{2}}{\partial s}-\varphi_{2} \tau_{g}+\varphi_{3} \kappa_{n} \\
& \frac{\partial \tau_{g}}{\partial t}=\frac{\partial \varphi_{3}}{\partial s}+\varphi_{1} \kappa_{n}-\varphi_{2} \kappa_{g}+2 \varphi_{3} \tau_{g} .
\end{aligned}
$$


Proof. It is well known that the arc length and time derivatives commute. This implies the inextensibility of $\gamma$. Accordingly, the compatibility conditions are $\frac{\partial}{\partial s}\left(\frac{\partial t}{\partial t}\right)=\frac{\partial}{\partial t}\left(\frac{\partial t}{\partial s}\right)$, etc. On the other hand,

$$
\begin{aligned}
\frac{\partial}{\partial s}\left(\frac{\partial \mathbf{t}}{\partial t}\right) & =\frac{\partial}{\partial s}\left(\varphi_{1} \mathbf{n}+\varphi_{2} \mathbf{g}\right) \\
& =\left(-\varphi_{1} \kappa_{n}-\varphi_{2} \kappa_{g}\right) \mathbf{t}+\left(\frac{\partial \varphi_{1}}{\partial s}+\varphi_{1} \tau_{g}\right) \mathbf{n}+\left(\frac{\partial \varphi_{2}}{\partial s}-\varphi_{2} \tau_{g}\right) \mathbf{g},
\end{aligned}
$$

and:

$$
\begin{aligned}
\frac{\partial}{\partial t}\left(\frac{\partial \mathbf{t}}{\partial s}\right) & =\frac{\partial}{\partial t}\left(\kappa_{g} \mathbf{n}+\kappa_{n} \mathbf{g}\right) \\
& =\left(-\varphi_{1} \kappa_{n}-\varphi_{2} \kappa_{g}\right) \mathbf{t}+\left(\frac{\partial \kappa_{g}}{\partial t}+\varphi_{3} \kappa_{g}\right) \mathbf{n}+\left(\frac{\partial \kappa_{n}}{\partial t}-\varphi_{3} \kappa_{n}\right) \mathbf{g} .
\end{aligned}
$$

Comparing the two equations, we find:

$$
\begin{aligned}
& \frac{\partial \kappa_{g}}{\partial t}=\frac{\partial \varphi_{1}}{\partial s}+\varphi_{1} \tau_{g}-\varphi_{3} \kappa_{g}, \\
& \frac{\partial \kappa_{n}}{\partial t}=\frac{\partial \varphi_{2}}{\partial s}-\varphi_{2} \tau_{g}+\varphi_{3} \kappa_{n} .
\end{aligned}
$$

It follows from (8) that we can obtain the first and the second equation of (12).

Furthermore by using $\frac{\partial}{\partial s}\left(\frac{\partial \mathbf{n}}{\partial t}\right)=\frac{\partial}{\partial t}\left(\frac{\partial \mathbf{n}}{\partial s}\right)$ and following a similar way as above, we can obtain the third equation of (12). The proof is completed.

Remark 1. As applications of inextensible flows of a spacelike curve on a lightlike surface, we can consider geometric phases of the repulsive-type nonlinear Schödinger equation $\left(N L S^{-}\right)$(cf. [12]).

\section{Inextensible Flows of a Lightlike Curve}

Let $\gamma$ be a lightlike curve on a lightlike surface $M$ in $\mathbb{R}_{1}^{3}$. We note that a lightlike curve $\gamma(u)$ satisfies $\left\langle\gamma^{\prime \prime}(u), \gamma^{\prime \prime}(u)\right\rangle \geq 0$. We say that a lightlike curve $\gamma(u)$ is parametrized by the pseudo arc length if $\left\langle\gamma^{\prime \prime}(u), \gamma^{\prime \prime}(u)\right\rangle=1$. If a lightlike curve $\gamma(u)$ satisfies $\left\langle\gamma^{\prime \prime}(u), \gamma^{\prime \prime}(u)\right\rangle \neq 0$, then $\left\langle\gamma^{\prime \prime}(u), \gamma^{\prime \prime}(u)\right\rangle>0$, and:

$$
s(u)=\int_{0}^{u}\left\langle\gamma^{\prime \prime}(u), \gamma^{\prime \prime}(u)\right\rangle^{\frac{1}{4}} d u
$$

becomes the pseudo arc length parameter. Let us consider a lightlike curve $\gamma(u)$ on a lightlike surface $M$ in $\mathbb{R}_{1}^{3}$ with $\left\langle\gamma^{\prime \prime}(u), \gamma^{\prime \prime}(u)\right\rangle \neq 0$.

Let $\gamma:[0, l] \times[0, w] \rightarrow M \subset \mathbb{R}_{1}^{3}$ be a one-parameter family of smooth lightlike curves on a lightlike surface in $\mathbb{R}_{1}^{3}$, where $l$ is the arc length of the initial curve. We put $v^{4}=\left\langle\gamma^{\prime \prime}(u), \gamma^{\prime \prime}(u)\right\rangle$, from which the pseudo arc length of $\gamma$ is defined by $s(u)=\int_{0}^{u} v d u$. Furthermore, the operator $\frac{\partial}{\partial s}$ is given in terms of $u$ by $\frac{\partial}{\partial s}=\frac{1}{v} \frac{\partial}{\partial u}$, and the pseudo arc length parameter is given by $d s=v d u$.

On the other hand, a flow $\frac{\partial \gamma}{\partial t}$ of $\gamma$ can be given by:

$$
\frac{\partial \gamma}{\partial t}=f_{1} \mathbf{t}+f_{2} \mathbf{n}+f_{3} \mathbf{g}
$$

in terms of the Darboux frames $\{\mathbf{t}, \mathbf{n}, \mathbf{g}\}$ of the lightlike curve $\gamma$ on a lightlike surface $M$ in $\mathbb{R}_{1}^{3}$, where $f_{1}, f_{2}, f_{3}$ are scalar speeds of the lightlike curve $\gamma$, respectively. We put $s(u, t)=\int_{0}^{u} v d u$, it is called the pseudo arc length variation of $\gamma$. From this, we have the following condition:

$$
\frac{\partial}{\partial t} s(u, t)=\int_{0}^{u} \frac{\partial v}{\partial t} d u=0
$$

for all $u \in[0, l]$. 
Definition 2. A curve evolution $\gamma(u, t)$ and its flow $\frac{\partial \gamma}{\partial t}$ of a lightlike curve $\gamma$ in $\mathbb{R}_{1}^{3}$ are said to be inextensible if:

$$
\frac{\partial}{\partial t}\left\langle\frac{\partial^{2} \gamma}{\partial u^{2}}, \frac{\partial^{2} \gamma}{\partial u^{2}}\right\rangle^{\frac{1}{4}}=0
$$

Theorem 5. Let $M$ be a lightlike surface in Minkowski three-space $\mathbb{R}_{1}^{3}$ and $\{\mathbf{t}, \mathbf{n}, \mathbf{g}\}$ be the Darboux frames along a lightlike curve $\gamma$ on $M$. If $\frac{\partial \gamma}{\partial t}=f_{1} \mathbf{t}+f_{2} \mathbf{n}+f_{3} \mathbf{g}$ is a flow of $\gamma$ on a lightlike surface $M$, then we have the following equation:

$$
\frac{\partial v}{\partial t}=\frac{1}{2 v^{3}}\left[\left(\frac{\partial v}{\partial u}+v^{2} \kappa_{n}\right)\left(\frac{\partial \Phi_{2}}{\partial u}-v \kappa_{n} \Phi_{2}-v \kappa_{g} \Phi_{3}\right)+v^{2} \kappa_{g}\left(\frac{\partial \Phi_{3}}{\partial u}+v \kappa_{g} \Phi_{1}+v \tau_{g} \Phi_{2}\right)\right]
$$

where:

$$
\begin{aligned}
& \Phi_{1}=\frac{\partial f_{1}}{\partial u}+v f_{1} \kappa_{n}-v f_{3} \tau_{g}, \\
& \Phi_{2}=\frac{\partial f_{2}}{\partial u}-v f_{2} \kappa_{n}-v f_{3} \kappa_{g}, \\
& \Phi_{3}=\frac{\partial f_{1}}{\partial u}+v f_{1} \kappa_{g}+v f_{2} \tau_{g} .
\end{aligned}
$$

Proof. From the definition of a lightlike curve $\gamma$, we have $v^{4}=\left\langle\frac{\partial^{2} \gamma}{\partial u^{2}}, \frac{\partial^{2} \gamma}{\partial u^{2}}\right\rangle$. By differentiating $v^{4}$, we have:

$$
4 v^{3} \frac{\partial v}{\partial t}=\frac{\partial}{\partial t}\left\langle\frac{\partial^{2} \gamma}{\partial u^{2}}, \frac{\partial^{2} \gamma}{\partial u^{2}}\right\rangle=2\left\langle\frac{\partial^{2} \gamma}{\partial u^{2}}, \frac{\partial^{2}}{\partial u^{2}}\left(\frac{\partial \gamma}{\partial t}\right)\right\rangle
$$

On the other hand,

$$
\frac{\partial^{2} \gamma}{\partial u^{2}}=\frac{\partial}{\partial u}\left(\frac{\partial \gamma}{\partial u}\right)=\frac{\partial}{\partial u}(v \mathbf{t})=\left(\frac{\partial v}{\partial u}+v^{2} \kappa_{n}\right) \mathbf{t}+v^{2} \kappa_{g} \mathbf{g}
$$

and:

$$
\begin{aligned}
& \frac{\partial^{2}}{\partial u^{2}}\left(\frac{\partial \gamma}{\partial t}\right)=\frac{\partial^{2}}{\partial u^{2}}\left(f_{1} \mathbf{t}+f_{2} \mathbf{n}+f_{3} \mathbf{g}\right) \\
& =\left[\frac{\partial \Phi_{1}}{\partial u}+v \kappa_{n} \Phi_{1}-v \tau_{g} \Phi_{3}\right] \mathbf{t}+\left[\frac{\partial \Phi_{2}}{\partial u}-v \kappa_{n} \Phi_{2}-v \kappa_{g} \Phi_{3}\right] \mathbf{n}+\left[\frac{\partial \Phi_{3}}{\partial u}+v \kappa_{g} \Phi_{1}+v \tau_{g} \Phi_{2}\right] \mathbf{g} .
\end{aligned}
$$

Thus, (16) implies (15). This completes the proof.

Theorem 6. Let $\frac{\partial \gamma}{\partial t}=f_{1} \mathbf{t}+f_{2} \mathbf{n}+f_{3} \mathbf{g}$ be a flow of a lightlike curve $\gamma$ on a lightlike surface $M$ in $\mathbb{R}_{1}^{3}$. Then, the flow is inextensible if and only if:

$$
\left(\frac{\partial v}{\partial s}+v \kappa_{n}\right) \frac{\partial \Phi_{2}}{\partial s}+v \kappa_{g} \frac{\partial \Phi_{3}}{\partial s}=\left(\frac{\partial v}{\partial s}+v \kappa_{n}\right)\left(\kappa_{n} \Phi_{2}+\kappa_{g} \Phi_{3}\right)-v \kappa_{g}\left(\kappa_{g} \Phi_{1}+\tau_{g} \Phi_{2}\right) .
$$

Proof. Suppose that the flow of a lightlike curve $\gamma$ on $M$ is inextensible. By using (15) and $\frac{\partial}{\partial s}=\frac{1}{v} \frac{\partial}{\partial u}$, (14) gives (17). Conversely, by following a similar way as above, the proof is completed.

Next, we give the time evolution equations of the Darboux frame of a lightlike curve on a lightlike surface. 
Theorem 7. Let $\frac{\partial \gamma}{\partial t}=f_{1} \mathbf{t}+f_{2} \mathbf{n}+f_{3} \mathbf{g}$ be a flow of a lightlike curve $\gamma$ on a lightlike surface $M$ in $\mathbb{R}_{1}^{3}$. If the flow is inextensible, then a time evolution of the Darboux frame $\{\mathbf{t}, \mathbf{n}, \mathbf{g}\}$ along a curve $\gamma$ on a lightlike surface $M$ is given by:

$$
\frac{d}{d t}\left(\begin{array}{l}
\mathbf{t} \\
\mathbf{n} \\
\mathbf{g}
\end{array}\right)=\left(\begin{array}{ccc}
\frac{\Phi_{1}}{v} & 0 & \frac{\Phi_{3}}{v} \\
0 & -\frac{\Phi_{1}}{v} & \Theta \\
-\Theta & -\frac{\Phi_{3}}{v} & 0
\end{array}\right)\left(\begin{array}{l}
\mathbf{t} \\
\mathbf{n} \\
\mathbf{g}
\end{array}\right)
$$

where $\Theta=\left\langle\frac{\partial \mathbf{n}}{\partial t}, \mathbf{g}\right\rangle$.

Proof. The proof can be obtained by using a similar method of proof of Theorem 3 .

Theorem 8. Let $\frac{\partial \gamma}{\partial t}=f_{1} \mathbf{t}+f_{2} \mathbf{n}+f_{3} \mathbf{g}$ be a flow of a lightlike curve $\gamma$ on a lightlike surface $M$ in $\mathbb{R}_{1}^{3}$. Then, the time evolution equations of the functions $\kappa_{g}, \kappa_{n}$ and $\tau_{g}$ for the inextensible spacelike curve $\gamma$ are given by:

$$
\begin{aligned}
\frac{\partial \kappa_{g}}{\partial t} & =\frac{\partial}{\partial s}\left(\frac{1}{v} \Phi_{3}\right)+\frac{1}{v}\left(\kappa_{g} \Phi_{1}-\kappa_{n} \Phi_{3}\right), \\
\frac{\partial \kappa_{n}}{\partial t} & =\frac{\partial}{\partial s}\left(\frac{1}{v} \Phi_{1}\right)+\kappa_{g} \Theta-\frac{1}{v} \tau_{g} \Phi_{3}, \\
\frac{\partial \tau_{g}}{\partial t} & =\frac{\partial \Theta}{\partial s}+\kappa_{n} \Theta-\frac{1}{v} \tau_{g} \Phi_{1} .
\end{aligned}
$$

Proof. The proof can be obtained by using a similar method of proof of Theorem 4 .

\section{Lightlike Ruled Surfaces}

In this section, we investigate inextensible flows of ruled surfaces, in particular lightlike ruled surfaces in Minkowski three-space $\mathbb{R}_{1}^{3}$.

Let $I$ be an open interval on the real line $\mathbb{R}$. Let $\alpha$ be a curve in $\mathbb{R}_{1}^{3}$ defined on $I$ and $\beta$ a transversal vector field along $\alpha$. For an open interval $J$ of $\mathbb{R}$, we have the parametrization for $M$ :

$$
X(u, v)=\alpha(u)+v \beta(u), \quad u \in I, \quad v \in J .
$$

Here, $\alpha$ is called a base curve and $\beta$ a director vector field. In particular, the director vector field $\beta$ can be naturally chosen so that it is orthogonal to $\alpha$, that is $\left\langle\alpha^{\prime}, \beta\right\rangle=0$. It is well known that the ruled surface is developable if $\operatorname{det}\left(\alpha^{\prime} \beta \beta^{\prime}\right)$ is identically zero. A developable surface is a surface whose Gaussian curvature of the surface is everywhere zero.

On the other hand, the tangent vectors are given by:

$$
X_{u}=\frac{\partial X}{\partial u}=\alpha^{\prime}(u)+v \beta^{\prime}(u), \quad X_{v}=\frac{\partial X}{\partial v}=\beta(u),
$$

which imply that the coefficients of the first fundamental form of the surface are given by:

$$
\begin{aligned}
& E=\left\langle X_{u}, X_{u}\right\rangle=\left\langle\alpha^{\prime}, \alpha^{\prime}\right\rangle+2 v\left\langle\alpha^{\prime}, \beta^{\prime}\right\rangle+v^{2}\left\langle\beta^{\prime}, \beta^{\prime}\right\rangle, \\
& F=\left\langle X_{u}, X_{v}\right\rangle=0, \\
& G=\left\langle X_{v}, X_{v}\right\rangle=\langle\beta, \beta\rangle .
\end{aligned}
$$

Suppose that the ruled surface is lightlike. Then, we get $E=0$ or $G=0$.

First of all, we consider $E=0$; it implies that:

$$
\left\langle\alpha^{\prime}, \alpha^{\prime}\right\rangle=0, \quad\left\langle\alpha^{\prime}, \beta^{\prime}\right\rangle=0, \quad\left\langle\beta^{\prime}, \beta^{\prime}\right\rangle=0 .
$$

Thus, a base curve $\alpha$ is lightlike, and a director vector $\beta$ is constant or $\beta^{\prime}$ is lightlike. 
Case 1: If $\beta$ is constant, from $\left\langle\alpha^{\prime}, \beta\right\rangle=0, \beta$ is a lightlike vector or a spacelike vector. If $\beta$ is lightlike, there exists a smooth function $k$ such that $\beta=k \alpha^{\prime}$. This is a contradiction because $G=0$. If $\beta$ is spacelike as a constant vector, then the lightlike cylindrical ruled surface is parametrized by:

$$
X(u, v)=\alpha(u)+v \beta,
$$

where $\alpha$ is a lightlike curve and $\beta$ is a constant spacelike vector.

Case 2: Let $\beta^{\prime}$ be a lightlike vector. Since $\left\langle\alpha^{\prime}, \beta^{\prime}\right\rangle=0$, there exists a smooth function $k$ such that $\beta^{\prime}=k \alpha^{\prime}$. Thus, a lightlike non-cylindrical ruled surface is parametrized by:

$$
X(u, v)=\alpha(u)+v \beta(u),
$$

where $\alpha$ and $\beta$ satisfy the condition (20).

Next, we consider $G=\langle\beta, \beta\rangle=0$, since $\beta \neq \mathbf{0}$, a director vector $\beta$ must be lightlike. Furthermore, since $\left\langle\alpha^{\prime}, \beta\right\rangle=0, \alpha$ is a spacelike curve or a lightlike curve.

Case 1: If $\alpha$ is a spacelike curve, then a lightlike non-cylindrical ruled surface is parametrized by:

$$
X(u, v)=\alpha(u)+v \beta(u),
$$

where $\alpha$ is a spacelike curve and $\beta$ is a lightlike vector.

Case 2: Let $\alpha$ be a lightlike curve. Then, there exists a smooth function $k$ such that $\beta^{\prime}=k \alpha^{\prime}$, and a lightlike ruled surface as a tangent developable surface is parametrized by:

$$
X(u, v)=\alpha(u)+v k \alpha^{\prime}(u),
$$

where $\alpha$ and $\alpha^{\prime \prime}$ are a lightlike curve and a spacelike vector, respectively.

In [5], the authors gave the following:

Definition 3. A surface evolution $X(u, v, t)$ and its flow $\frac{\partial X}{\partial t}$ are said to be inextensible if the coefficients of the first fundamental form of the surface satisfy:

$$
\frac{\partial E}{\partial t}=\frac{\partial F}{\partial t}=\frac{\partial G}{\partial t}=0 .
$$

This definition states that the surface $X(u, v, t)$ is, for all time $t$, the isometric image of the original surface $X\left(u, v, t_{0}\right)$ defined at some initial time $t_{0}$.

Now, we study inextensible flows of a lightlike tangent developable surface in Minkowski three-space.

Consider a lightlike tangent developable surface parametrized by:

$$
X(u, v)=\alpha(u)+v \alpha^{\prime}(u),
$$

where $\alpha$ is a lightlike curve. Suppose that the parameter $u$ is a pseudo-arc length of $\alpha$. In this case, we get $E=v^{2}\left\|\alpha^{\prime \prime}\right\|^{2}$ and $F=G=0$.

Thus, we have:

Theorem 9. Let $X(u, v)$ be a lightlike tangent developable surface given by (24). The surface evolution $X(u, v, t)=\alpha(u, t)+v \alpha^{\prime}(u, t)$ is inextensible if and only if:

$$
\frac{\partial}{\partial t}\left\|\alpha^{\prime \prime}\right\|^{2}=0 .
$$

As a consequence, we have the following results: 
Theorem 10. Let $X(u, v, t)=\alpha(u, t)+v \alpha^{\prime}(u, t)$ be a surface evolution of a lightlike tangent developable surface given by (24) in $\mathbb{R}_{1}^{3}$. Then, we have the following statements:

(1) $\alpha(u, t)$ is an inextensible evolution of a lightlike curve $\alpha(u)$ in $\mathbb{R}_{1}^{3}$.

(2) An inextensible evolution of a lightlike tangent developable surface can be completely characterized by the inextensible evolutions of a lightlike curve $\alpha(u)$ in $\mathbb{R}_{1}^{3}$.

Proof. In fact, $0=\left.\frac{\partial}{\partial t}\left\|\alpha^{\prime \prime}\right\|\right|^{2}=2\left\|\alpha^{\prime \prime}\right\| \frac{\partial}{\partial t}\left\|\alpha^{\prime \prime}\right\|$ and $\alpha^{\prime \prime} \neq 0$, and we get $\frac{\partial}{\partial t}\left\|\alpha^{\prime \prime}\right\|=0$; it implies $\frac{\partial}{\partial t}\left\|\alpha^{\prime \prime}\right\| \frac{1}{2}=0$. This means that $\alpha(u, t)$ satisfies the condition for Definition 2 .

Theorem 11. Let $X(u, v, t)=\alpha(u, t)+v \alpha^{\prime}(u, t)$ be a surface evolution of a lightlike tangent developable surface given by (24) in $\mathbb{R}_{1}^{3}$, and $\frac{\partial \alpha}{\partial t}=f_{1} \mathbf{t}+f_{2} \mathbf{n}+f_{3} \mathbf{g}$, where $\mathbf{t}, \mathbf{n}, \mathbf{g}$ are the Darboux frames along $a$ lightlike curve $\alpha$ on a lightlike surface. If the surface evolution $X(u, v, t)$ is inextensible, then $f_{1}, f_{2}, f_{3}$ satisfy Equation (19).

\section{Conclusions}

We study an inextensible flow of a spacelike or a lightlike curve on a lightlike surface in Minkowski three-space and investigate a time evolution of the Darboux frame $\{\mathbf{t}, \mathbf{n}, \mathbf{g}\}$ (see Theorems 3 and 7) and the functions $\kappa_{n}, \kappa_{g}$ and $\tau_{g}$ (see Theorems 4 and 8). Furthermore, in Theorems 2 and 6, we give a necessary and sufficient condition of inextensible flows of a spacelike curve and a lightlike curve on a lightlike surface in terms of a partial differential equation involving the curvatures of the curve on a lightlike surface. Finally, we completely classify lightlike ruled surfaces in Minkowski three-space and characterize an inextensible evolution of a lightlike curve on a lightlike tangent developable surface (see Theorems 9 and 10).

Author Contributions: D.W.Y. gave the idea of inextensible flows of a spacelike curve and a lightlike curve on a lightlike surface. Z.K.Y. checked and polished the draft.

Funding: The second author was supported by the Basic Science Research Program through the National Research Foundation of Korea (NRF) funded by the Ministry of Education (NRF-2018R1D1A1B07046979).

Conflicts of Interest: The authors declare no conflict of interest.

\section{References}

1. Chirikjian, G.S.; Burdick, J.W. Kinematics of hyper-redundant manipulation. In Proceedings of the ASME Mechanisms Conference, Chicago, IL, USA, 16-19 September 1990; pp. 391-396.

2. Desbrun, M.; Cani-Gascuel, M.-P. Active implicit surface for animation. In Graphics Interface; The Canadian Information Processing Society: Mississauga, ON, Canada, 1998; pp. 143-150.

3. Kass, M.; Witkin, A.; Terzopoulos, D. Snakes: Active contour models. In Proceedings of the 1st International Conference on Computer Vision, London, UK, 8-11 June 1987; pp. 259-268.

4. Schief, W.K.; Rogers, C. Binormal motion of curves of constant curvature and torsion. generation of soliton surfaces. Proc. R. Soc. Lond. A 1999, 455, 3163-3188. [CrossRef]

5. Kwon, D.Y.; Park, F.C. Inextensible flows of curves and developable surfaces. Appl. Math. Lett. 2005, 18, 1156-1162. [CrossRef]

6. Mohamed, S.G. Binormal motions of inextensible curves in de-sitter space $\mathbb{S}^{2,1}$. J. Egypt. Math. Soc. 2017, 25, 313-318. [CrossRef]

7. Andrews, B. Classification of limiting shapes for isotropic curve flows. J. Am. Math. Soc. 2003, 16, 443-459. [CrossRef]

8. Gurbuz, N. Inextensible flows of spacelike, timelike and null curves. Int. J. Contemp. Math. Sci. 2009, 4, 1599-1604.

9. Hussien, R.A.; Mohamed, S.G. Generated surfaces via inextensible flows of curves in $\mathbb{R}^{3}$. J. Appl. Math. 2016, 2016, 6178961. [CrossRef]

10. Yeneroglu, M. On new characterization of inextensible flows of space-like curves in de Sitter space. Open Math. 2016, 14, 946-954. [CrossRef] 
11. Zhu, X.-P. Asymptotic behavior of anisotropic curve flows. J. Differ. Geom. 1998, 48, 225-274. [CrossRef]

12. Gurbuz, N. Three clasess of non-lightlike curve evolution according to Darboux frame and geometric phase. Int. J. Geom. Methods Mod. Phys. 2018, 15, 1850023. [CrossRef]

(C) 2018 by the authors. Licensee MDPI, Basel, Switzerland. This article is an open access article distributed under the terms and conditions of the Creative Commons Attribution (CC BY) license (http://creativecommons.org/licenses/by/4.0/). 